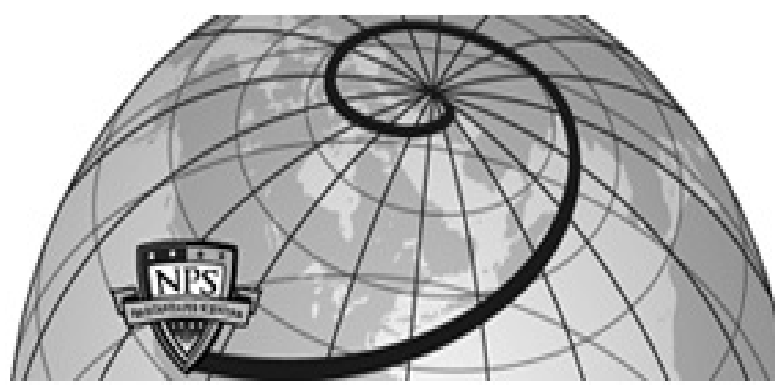

Calhoun: The NPS Institutional Archive DSpace Repository

\title{
Pseudospectral Optimal Control for Military and Industrial Applications
}

Gong, Qi; Kang, Wei; Bedrossian, Nazareth S.; Fahroo, Fariba; Sekhavat, Pooya; Bollino, Kevin; Lewis, Ryan IEEE

https://hdl.handle.net/10945/29677

This publication is a work of the U.S. Government as defined in Title 17, United States Code, Section 101. Copyright protection is not available for this work in the United States.

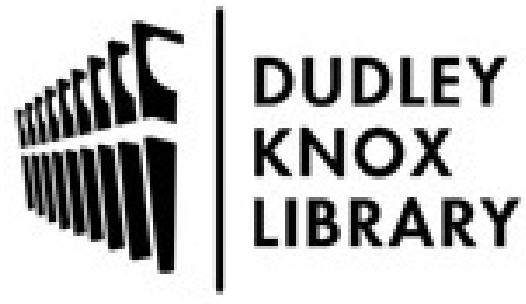

http://www.nps.edu/library
Calhoun is the Naval Postgraduate School's public access digital repository for research materials and institutional publications created by the NPS community. Calhoun is named for Professor of Mathematics Guy K. Calhoun, NPS's first appointed -- and published -- scholarly author.

Dudley Knox Library / Naval Postgraduate School 411 Dyer Road / 1 University Circle Monterey, California USA 93943 


\title{
Pseudospectral Optimal Control for Military and Industrial Applications
}

\author{
Qi Gong, Wei Kang, Nazareth S. Bedrossian, Fariba Fahroo, Pooya Sekhavat and Kevin Bollino
}

\begin{abstract}
During the last decade, pseudospectral methods for optimal control, the focus of this tutorial session, have been rapidly developed as a powerful tool to enable new applications that were previously considered impossible due to the complicated nature of these problems. The purpose of this tutorial section is to introduce this advanced technology to a wider community of control system engineering. We bring in experts of pseudospectral methods from academia, industry, and military and DoD to present topics covering a large spectrum of pseudospectral methods, including the theoretical foundation, numerical techniques of pseudospectral optimal control, and military/industry applications.
\end{abstract}

Over the last few years, pseudospectral (PS) methods for solving optimal control problems have moved rapidly from mathematical theory to real-world applications. For example, on November 5, 2006, and March 3, 2007, by tracking an attitude trajectory developed with pseudospectral optimal control theory, the International Space Station (ISS) completed two large angle maneuvers without using any propellant. In addition to saving NASA $\$ 1.5$ million in propellant cost, this is the first time that zero-propellant maneuver is successfully carried out for large angle rotations, a mission impossible by using the current ISS control software that is based on eigenaxis trajectories. The success of pseudospectral methods is a result of recent advances in theory, algorithms, and computational power. These advances in algorithms and technologies make it possible to solve highly complicated nonlinear optimal control problems in real-life applications. The purpose of this special session is to bring together leading experts on PS optimal control to outline the rapid advances from theory to practice.

In addition to the flight demonstration of PS optimal control of ISS by Dr. Bedrossian of Draper Labs at NASA, Houston, the session includes a presentation by Dr. Fahroo of AFOSR who will present a unified view on discrete optimality conditions for PS methods. A real-life demonstration of these optimality conditions will he illustrated by Dr. Sekhavat of US Naval Postgraduate School by way of optimal feed-

This research was supported by AFOSR under grant F1ATA0-60-6$2 \mathrm{G} 002$.

Dept. of Elec. \& Comp. Engr., Univ. of Texas at San Antonio, qi.gong@utsa.edu

Dept. of Applied Math., Naval Postgraduate School, Monterey, CA, 93943, wkang@nps. edu

The Charles Stark Draper Laboratory, Inc., Houston, TX 77058 naz@jsc.draper.com

AFOSR Program Manager, Computational Mathematics, Arlington, VA 22203, fariba.fahroolafosr.af.mil

Dept. of Mech. \& Astro. Eng., Naval Postgraduate School, Monterey, CA 93943, psekhava@nps.edu

Air Force Element of Space Technology, Washington DC, 20003, bollinok@verizon.net back control of NPSAT1, an experimental military spacecraft scheduled to be launched in 2009. Optimal motion planning for autonomous vehicles in obstacle rich environments will be the subject of the talk by Dr. Gong of University of Texas at San Antonio. Maj. Bollino, USAF, will present applications of PS methods for an AFRL project on generating realtime optimal trajectories for reusable launch vehicles. He will demonstrate the robustness of a PS guidance method to ensure the landing of an X-33 type vehicle in an uncertain environment. The leading presentation of the special session is an overview of pseudospectral methods to be given by Wei Kang of US Naval Postgraduate School. It includes a brief literature of general pseudospectral approximations, the theoretical foundation of the pseudospectral optimal control, the interplay between discrete approximations and the various engineering applications, and the advantages as well as challenges of pseudospectral optimal control methods.

In addition to the applications in these invited talks, PS optimal controllers have been extensively used to solve a wide range of problems such as those arising in UAV trajectory generation, missile guidance, control of robotic arms, vibration damping, lunar guidance, magnetic control, swingup and stabilization of an inverted pendulum, orbit transfers, tether libration control, and ascent guidance. These solutions have largely been facilitated by two software packages: DIDO [34] and OTIS [29]. DIDO is a commercially available MATLAB software package while OTIS was developed by Boeing for NASA.

The topics of this special session cover pseudospectral optimal control applications ranging from space to ground, as well as advanced computational and theoretical results that have been discovered only recently. Given the rapid rise of PS controllers, we believe many more research areas and engineering applications can be benefited by this innovative method. This special session provides a unique opportunity to CDC attendees to engage in the enabling technology of pseudospectral methods.

\section{Pseudospectral Optimal Control for CONSTRAINEd NONLINEAR PROBLEMS - WeI KANG AND QI Gong}

\section{A. Introduction}

A fundamental problem in autonomous systems engineering is the computation of constrained nonlinear optimal controls. Since the 1960s, many computational methods have been proposed toward the goal of providing robust and accurate algorithms for solving these problems. Over the last decade, a computational approach based on discrete approximations has gained wide popularity as a result of significant 
progress in large-scale computation and the robustness of the approach, see [19], [2], [7], [8], [18] just to name a few. The essential idea of this method is to discretize the optimal control problem and then solve the resulting largescale finite-dimensional optimization problem. The roots of these methods can be traced back to the works of Bernoulli and Euler [33]. The simplicity of this approach belies a wide range of deep theoretical issues (see [28]) that lie at the intersection of approximation theory, control theory and optimization. Even though these issues are yet be satisfactorily addressed and dealt with, a wide variety of industrial-strength optimal control problems have already been solved by this approach [2], [19], [20], [27], [36], [44].

In this presentation we focus on pseudospectral (PS) methods. PS methods were largely developed in the 1970s for solving partial differential equations arising in fluid dynamics and meteorology [3], and quickly became "one of the big three technologies for the numerical solution of PDEs" [45]. During the 1990s, PS methods were introduced for solving optimal control problems; and since then, have gained considerable attention [8], [9], [20], [21], [27], [41], [31], [44], [48], [49], particularly in solving aerospace control problems. Examples range from lunar guidance [20], magnetic control [49], orbit transfers [44], tether libration control [48], ascent guidance [27] and a host of other problems. Recently, PS optimal control methods have been applied to the attitude control of International Space Station. on November 5, 2006, and March 3, 2007, by tracking an attitude trajectory developed with pseudospectral optimal control theory, ISS completed two large angle maneuvers without using any propellant. This is the first successful zeropropellant maneuver of ISS in large angle rotations. As a result of its success, PS methods are now part of OTIS [29], NASA's software package for solving trajectory optimization problems. In addition, the commercially available software package, DIDO [34], exclusively uses PS methods for solving optimal control problems.

\section{B. Pseudospectral Methods for Approximation}

What makes pseudospectral methods so attractive? PS methods were originally developed for solving partial differential equations. In the discretization of a PDE, the continuous functions are approximated at a finite set of nodes. These nodes are carefully selected to achieve high accuracy in approximation. Then, the PDE is discretized and the equation is approximated by a set of ODEs enforced at the node points. A signature of the PS method is its clever way of discretization. For a brief illustration of the basic ideas, let us consider a real valued function $f(t)$ defined on an interval $[a, b]$. A straightforward way of approximation is to use interpolation based upon its value at equal distance nodes:

$$
t_{0}=a, t_{1}=(b-a) / N, t_{2}=2(b-a) / N, \cdots, t_{N}=b
$$

However, it was proved in numerical analysis that this simple way of node selection is not efficient; and more sophisticated node selection methods are able to achieve significantly improved accuracy with much less number of nodes. For example, a set of nodes located at the zeros of the derivatives of Legendre polynomials is called Legendre-Gauss-Lobatto nodes, or simply LGL nodes. An example of LGL nodes with $N=16$ is shown in Figure 1. Let $I_{N} f(t)$ denote the

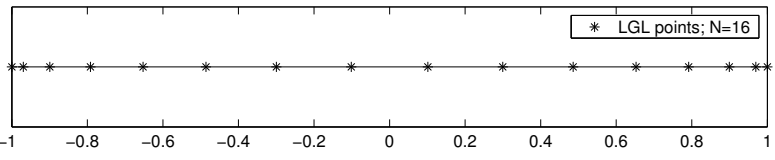

Fig. 1. LGL nodes $N=16$

polynomial interpolation of $f(t)$ at the LGL nodes in the interval $[-1,1]$. The following inequality is proved in the literature of spectral methods,

$$
\left\|f(t)-I_{N} f(t)\right\|_{L^{2}} \leq \frac{C}{N^{m}}
$$

where $m$ is the smoothness of $f(t)$ and $C$ is a constant independent of $N$. As $N \rightarrow \infty$, the polynomial interpolation at the LGL nodes converges to $f(t)$ under the $L^{2}$ norm at a rate of $1 / N^{m}$. Note if $f(t)$ is $C^{\infty}$, then $m=\infty$. This implies that the polynomial interpolation at the LGL nodes converges at a spectral rate, i.e it is faster than any given polynomial rate.

From a viewpoint of numerical analysis, this is an extremely impressive convergence rate. This fast convergence rate is especially attractive for solving optimal control problems. In optmial control, the rate of convergence is not merely an issue of efficiency; more importantly it is about feasibility. An increased number of nodes in the discretization of an optimal control problem results in a higher dimension in the nonlinear programming problem; and it increases the complexity in the searching for the optimal solution. A computational method becomes practically infeasible when the dimension and complexity of the nonlinear programming exceed the available computational power.

Solving the problem of optimal control requires the approximation of three types of mathematical objects: the integration in the cost function, the differential equation of the control system, and the state-control constraints. An ideal approximation method should be efficient for all the three approximation tasks. A method that is efficient for one of them, for instance an efficient ODE solver, may not be an efficient method for the other two objects. These requirements make the PS method an ideal approach because it is a proved fact in the literature that PS method is efficient for the approximation of all the three mathematical objects. In our approach, we use quadrature rule to approximate the integral, which achieves zero error integration for polynomial integrands of degree less than or equal to $2 N-1$. In the discretization of the ODE, a simple differentiation matrix is used for the derivatives. Because a PS method enforces the system at the selected nodes, the state-control constraints can be discretized straightforwardly. Overall, PS optimal control is a best all-round method for complicated nonlinear problems of optimal control. 


\section{Pseudospectral Optimal Control Methods}

In the following we summarize the outline of pseudospectral nonlinear optimal control methods; details can be found in [9], [12], [14], [22]. Consider the following nonlinear constrained optimal control problem:

Problem B: Determine the state-control function pair, $t \mapsto$ $(x, u) \in \mathbb{R}^{N_{x}} \times \mathbb{R}^{N_{u}}$, that minimizes the cost function

$$
J[x(\cdot), u(\cdot)]=\int_{-1}^{1} F(x(t), u(t)) d t+E(x(-1), x(1))
$$

subject to the dynamics $\dot{x}(t)=f(x(t), u(t))$; endpoint conditions $e(x(-1), x(1))=0$; and path constraints $h(x(t), u(t)) \leq 0$.

It is assumed that $F: \mathbb{R}^{N_{x}} \times \mathbb{R}^{N_{u}} \rightarrow \mathbb{R}, E: \mathbb{R}^{N_{x}} \times \mathbb{R}^{N_{x}} \rightarrow$ $\mathbb{R}, f: \mathbb{R}^{N_{x}} \times \mathbb{R}^{N_{u}} \rightarrow \mathbb{R}^{N_{x}}, e: \mathbb{R}^{N_{x}} \times \mathbb{R}^{N_{x}} \rightarrow \mathbb{R}^{N_{e}}$, and $h: \mathbb{R}^{N_{x}} \times \mathbb{R}^{N_{u}} \rightarrow \mathbb{R}^{N_{h}}$, are continuously differentiable with respect to their arguments and their gradients are Lipschitz continuous over the domain. In order to apply the first order necessary conditions, appropriate constraint qualifications are implicitly assumed. In addition to these standard assumptions, we assume that an optimal solution $\left(x^{*}(\cdot), u^{*}(\cdot)\right)$ exists with the optimal state, $x^{*}(\cdot)$ in the Sobolev space $\in W^{m, \infty}, m \geq 1$.

We take the Legendre PS method as an example while the results are applicable to other PS methods. The state and control functions, $x(t)$ and $u(t)$, are approximated by $N$-th order Lagrange polynomials based on the interpolation at the LGL quadrature nodes. The LGL nodes, $t_{0}=-1<t_{1}<$ $\cdots<t_{N}=1$, are defined by

$$
t_{0}=-1, t_{N}=1 \text {, and }
$$

for $k=1,2, \ldots, N-1, t_{k}$ are the roots of $\dot{L}_{N}(t)$

where $\dot{L}_{N}(t)$ is the derivative of the $N$-th order Legendre polynomial $L_{N}(t)$. In the discretization, the state variables are approximated by the vectors $\bar{x}^{N k} \in \mathbb{R}^{\mathrm{r}}$, i.e.

$$
\bar{x}^{N k}=\left[\begin{array}{llll}
\bar{x}_{1}^{N k} & \bar{x}_{2}^{N k} & \ldots & \bar{x}_{r}^{N k}
\end{array}\right]^{T}
$$

is an approximation of $x\left(t_{k}\right)$. Similarly, $\bar{u}^{N k}$ is the approximation of $u\left(t_{k}\right)$. Thus, a discrete approximation of the function $x_{i}(t)$ is the vector

$$
\bar{x}_{i}^{N}=\left[\begin{array}{llll}
\bar{x}_{i}^{N 1} & \bar{x}_{i}^{N 2} & \cdots & \bar{x}_{i}^{N N}
\end{array}\right]
$$

A continuous approximation is defined by its polynomial interpolation, denoted by $x_{i}^{N}(t)$, i.e.

$$
x_{i}(t) \approx x_{i}^{N}(t)=\sum_{k=0}^{N} \bar{x}_{i}^{N k} \phi_{k}(t),
$$

where $\phi_{k}(t)$ is the Lagrange interpolating polynomial. In the notations, the discrete variables are denoted by letters with an upper bar, such as $\bar{x}_{i}^{N k}$ and $\bar{u}^{N k}$. If $k$ in the superscript and/or $i$ in the subscript are missing, it represents the corresponding vector or matrix in which the indices run from minimum to maximum. For example,

$$
\bar{x}^{N}=\left[\begin{array}{cccc}
\bar{x}_{1}^{N 0} & \bar{x}_{1}^{N 1} & \ldots & \bar{x}_{1}^{N N} \\
\bar{x}_{2}^{N 0} & \bar{x}_{2}^{N 1} & \ldots & \bar{x}_{2}^{N N} \\
\vdots & \vdots & \vdots & \vdots \\
\bar{x}_{r}^{N 0} & \bar{x}_{r}^{N 1} & \cdots & \bar{x}_{r}^{N N}
\end{array}\right]
$$

The derivative of $x_{i}^{N}(t)$ at the LGL node $t_{k}$ is easily computed by the following matrix multiplication

$$
\left[\begin{array}{llll}
\dot{x}_{i}^{N}\left(t_{0}\right) & \dot{x}_{i}^{N}\left(t_{1}\right) & \cdots & \dot{x}_{i}^{N}\left(t_{N}\right)
\end{array}\right]^{T}=D\left(\bar{x}_{i}^{N}\right)^{T}
$$

where the $(N+1) \times(N+1)$ differentiation matrix $D$ is defined by

$$
D_{i k}= \begin{cases}\frac{L_{N}\left(t_{i}\right)}{L_{N}\left(t_{k}\right)} \frac{1}{t_{i}-t_{k}}, & \text { if } i \neq k \\ -\frac{N(N+1)}{4}, & \text { if } i=k=0 \\ \frac{N(N+1)}{4}, & \text { if } i=k=N \\ 0, & \text { otherwise }\end{cases}
$$

The cost functional $J[x(\cdot), u(\cdot)]$ is approximated by the Gauss-Lobatto integration rule,

$$
\begin{aligned}
J[x(\cdot), u(\cdot)] \approx \bar{J}^{N}\left(\bar{x}^{N}, \bar{u}^{N}\right)= & \sum_{k=0}^{N} F\left(\bar{x}^{N k}, \bar{u}^{N k}\right) w_{k} \\
& +E\left(\bar{x}^{N 0}, \bar{x}^{N N}\right)
\end{aligned}
$$

where $w_{k}$ are the LGL weights defined by

$$
w_{k}=\frac{2}{N(N+1)} \frac{1}{\left[L_{N}\left(t_{k}\right)\right]^{2}},
$$

Now, we can define the discretization of Problem B. Let $\mathbb{X}$ and $\mathbb{U}$ be two compact sets representing the search region.

Problem $\mathbf{B}^{\mathbf{N}}$ : Find $\bar{x}^{N k} \in \mathbb{X}$ and $\bar{u}^{N k} \in \mathbb{U}, k=$ $0,1, \ldots, N$, that minimize

$$
\bar{J}^{N}\left(\bar{x}^{N}, \bar{u}^{N}\right)=\sum_{k=0}^{N} F\left(\bar{x}^{N k}, \bar{u}^{N k}\right) w_{k}+E\left(\bar{x}^{N 0}, \bar{x}^{N N}\right)
$$

subject to

$$
\begin{aligned}
\left\|\sum_{j=0}^{N} D_{k j} \bar{x}^{N j}-f\left(\bar{x}^{N k}, \bar{u}^{N k}\right)\right\|_{\infty} & \leq(N-1)^{\frac{3}{2}-m} \\
\left\|e\left(\bar{x}^{N 0}, \bar{x}^{N N}\right)\right\|_{\infty} & \leq(N-1)^{\frac{3}{2}-m} \\
h\left(\bar{x}^{N k}, \bar{u}^{N k}\right) & \leq(N-1)^{\frac{3}{2}-m} \cdot \mathbf{1}
\end{aligned}
$$

This optimization problem can be solved by taking the advantage of existing methods and software of nonlinear programming. In practice, a sequence of nonlinear programming problems with increasing number of nodes are obtained by successive mesh refinements [15] and the last problem is declared as the solution to meet the requested tolerance. For many examples, these sequence of problems can be solved in fractions of a second as a result of the covector mapping principle (CMP) [9], [33], [14] which facilitates a hot-start for the problem in the next sequence. 


\section{Theoretical Foundation}

The simplicity of pseudospectral methods masks a wide range of deeply theoretical issues that lie at the intersection of approximation theory and control theory. In an integrated computational framework, the convergence of an algorithm is essential. It can be shown that some popular RungeKutta methods that are convergent for initial value problems are non-convergent for optimal control [18]. On the other hand, it was found in [1] that non-convergent Runge-Kutta methods might converge for optimal control problems. Thus, the convergence of discretization methods for optimal control problems continues to be a topic of active research [1], [18], [7], [6].

Discrete approximations of optimal control problems pose many theoretical problems that are deceptively simple. For example, does the discretized problem always admit a feasible trajectory? Does a sequence of discretized optimal solutions converge to the continuous-time optimal solution? These questions are of interest not only from a theoretical standpoint, but are also of great practical value, particularly in the real-time computation of optimal control [35].

In the following we summarize some recent results regarding the feasibility and convergence of the PS optimal control methods. They provide a solid theoretical foundation for developing robust and efficient nonlinear optimal control solver.

Theorem 1: [13], [14][Existence] Suppose Problem $B$ has a feasible trajectory, $t \mapsto(x, u)$, in which $x(\cdot) \in W^{m, \infty}$ with $m \geq 2$. Then, there exists a positive integer $N_{1}$ such that, for any $N>N_{1}$, the feasible set of relaxed discretized problem (Problem $B^{N}$ ) is nonempty.

The result theoretically guarantees the well-poseness of the practical implementation of pseudospectral computational optimal control methods. By this result, the feasible set of Problem $\mathrm{B}^{\mathrm{N}}$ is nonempty as long as a sufficient number of nodes are used. Therefore, an optimal solution always exists. Note that the relaxation in the discretized dynamics and path constraints, (4)-(6), is essential to guarantee the feasibility, since there are counter examples [12] showing that if not properly relaxed the discretized problem may have no feasible trajectory. This problem is not unique to pseudospectral methods. Even for Euler discretization of optimal control problems, relaxation is necessary to guarantee the feasibility [5].

Although Theorem 1 is revealing, it does not yet complete the practical foundation in solving the optimal control problem since we need a connection between a discretized solution and the optimal solution. This connection was obtained in [12], [13] for the Legendre PS methods under a consistency assumption, a basic assumption in Polak's theory of consistent approximations [30]. For the reason of space, we briefly introduce one result in [13] only.

Let $\left(\bar{x}^{* N}, \bar{u}^{* N}\right)$ be a sequence of optimal solutions to Problem $\mathrm{B}^{\mathrm{N}}$. Let $x^{N}(t) \in \mathbb{R}^{N_{x}}$ be the $N$-th order interpolating polynomial of $\left(\bar{x}^{* N 0}, \ldots, \bar{x}^{* N N}\right)$ and $u^{N}(t) \in \mathbb{R}^{N_{u}}$ be any interpolation of $\left(\bar{u}^{* N 0}, \ldots, \bar{u}^{* N N}\right)$, i.e.

$$
x^{N}(t)=\sum_{k=0}^{N} \bar{x}^{* N k} \phi_{k}(t), \quad u^{N}(t)=\sum_{k=0}^{N} \bar{u}^{* N k} \psi_{k}(t)
$$

where $\phi_{k}(t)$ is the Lagrange interpolating polynomial and $\psi_{k}(t)$ is any continuous function such that $\psi_{k}\left(t_{j}\right)=1$, if $k=j$ and $\psi_{k}\left(t_{j}\right)=0$, if $k \neq j$. Note that $u^{N}(t)$ is not necessarily a polynomial. Typically, we use linear or spline functions for interpolating $\left(\bar{u}^{* N 0}, \ldots, \bar{u}^{* N N}\right)$. Now we consider the convergence of the sequences of discrete optimal solutions $\left\{\left(\bar{x}^{* N}, \bar{u}^{* N}\right)\right\}_{N=N_{1}}^{\infty}$ and their interpolating functions $\left\{x^{N}(t), u^{N}(t)\right\}_{N=N_{1}}^{\infty}$.

Definition 1: A continuous function $\rho(t)$ is called a uniform accumulation point of a function sequence $\left\{\rho^{N}(t)\right\}_{N=0}^{\infty}, t \in[-1,1]$, if there exists a subsequence of $\left\{\rho^{N}(t)\right\}_{N=0}^{\infty}$ that uniformly converges to $\rho(t)$.

Assumption 1: Assume $\left\{\bar{x}^{* N 0}, \dot{x}^{N}(t), u^{N}(t)\right\}_{N=N_{1}}^{\infty}$ has an accumulation point $\left(x_{0}^{\infty}, q(t), u^{\infty}(t)\right)$.

Theorem 2: Suppose Problem $B$ has an optimal solution $\left(x^{*}(t), u^{*}(t)\right)$ in which $x^{*}(t) \in W^{m, \infty}$ with $m \geq 2$. Let $\left\{\left(\bar{x}^{* N}, \bar{u}^{* N}\right)\right\}_{N=N_{1}}^{\infty}$ be a sequence of optimal solutions of Problem $\mathrm{B}^{\mathrm{N}}$ satisfying Assumption 1. Then, $u^{\infty}(t)$ is an optimal control to the original continuous Problem $\mathrm{B}$, and $x^{\infty}(t)=\int_{-1}^{t} q(\tau) d \tau+x_{0}^{\infty}$ is the corresponding optimal trajectory.

This result demonstrates a key property of PS discretization methods: if the optimal solution of the discrete Problem $B^{N}$ converges, it must converge to an optimal solution of the continuous Problem B. Thus, under relatively mild conditions, Theorem 1-2 guarantee the existence and convergence of the discrete-time optimal solution to the continuoustime solution of the original problem. Recently, significant progress has been made in the existence and convergence theory. In [22], the existence and convergence theorems without the consistency assumption are proved for feedback linearizable control systems, a first result beyond the consistent approximation theory for PS optimal control methods. In addition, the existence and convergence theorems for discontinuous optimal controls are proved in [24] and [23].

Applying similar arguments to the necessary conditions of the continuous optimal control problems (minimum principle), and the necessary condition of discrete optimization problems (KKT conditions), the costate information can be recovered from the discrete KKT multipliers. This part of results are summarized as a Covector Mapping Theorem in [13], [14]. Thus without deriving complicated continuous optimal necessary conditions, both primal and dual variables can be obtained by pseudospectral methods (see Figure 2 for illustration). The information of the dual variable can then be used for verification of the optimality or sensitivity analysis as well as warm start technique to speed up the computation.

A main drawback of the above theory on consistency approximation is Assumption 1, which is difficult to verify. Some significant progresses have been made recently on a selfcontained convergence theory that do not rely on Assumption 1. In [25], a theorm on the convergence of PS 


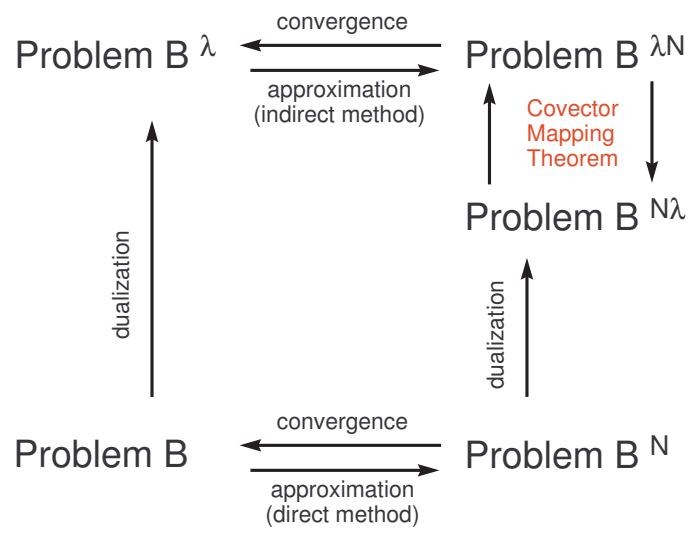

Fig. 2. Schematic for the Covector Mapping Principle [39], [40], [33].

optimal control methods for feedback linearizable systems is proved. This paper contains several essential differences from existing papers on PS optimal control as well as some other direct computational methods. The proof does not use the necessary conditions of optimal control or Pontryagin's minimum principle. Furthermore, the theory does not make coercivity type of assumptions. As a result, it does not require the local uniqueness of optimal solutions. Therefore, it is applicable to problems with multiple optimal solutions that exist in a small neighborhood. The most restrictive assumptions on consistency approximation made in [12] and [13] are removed from the theory. The key that makes these differences possible is that we introduce a set of sophisticated constraints in the discretization so that the computational algorithm has a greater control of the boundedness of the approximate solutions and their derivatives. From a geometric view point, these constraints reshape the boundaries of the search-region for the discretized nonlinear optimization problem. Therefore, different from the existing results in the literature of direct methods of optimal control, the desired boundedness is achieved not by making harsh assumptions on the original problem, but by implementing carefully designed shape of search-region for the discrete problem of nonlinear optimization.

\section{E. Closed-loop Implementation}

The Holy Grail in control theory and engineering is feedback closed-loop solution. However, except for special cases like linear-quadratic problems, no analytical solutions have been found for general constrained nonlinear optimal control problems. The difficulties stem from the challenges in solving the associated Hamilton-Jacobi-Bellman (HJB) equation which suffers by well-known curse of dimensionality. An alternative to solving the HJB equation is to seek online and real-time open loop solutions. This simple concept circumvents the difficulties associated with the HJB equation but relies heavily on modern computational capabilities to generate real-time solutions.

1) Closed-loop structures: Due to the fast convergence properties [12], [13], PS methods are capable of generating optimal solutions in a time efficiently fashion even for complicated nonlinear systems [35]; therefore, feedback through real-time optimal control is possible by way of non-analytical output-to-input maps [37]. Based on these enabling technologies, different feedback mechanisms can be formulated. In this section we briefly explain some of the closed-loop implementations of pseudospectral optimal control that have been applied in solving engineering applications.

Figure 3 demonstrates an inner-outer loop structure for achieving optimal feedback control. Under this structure,

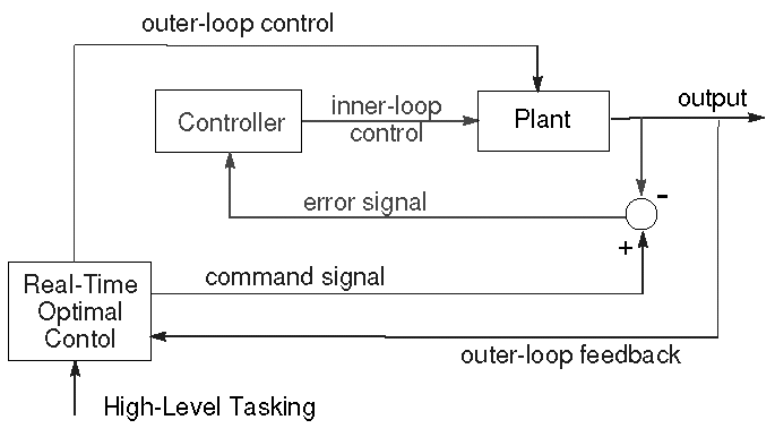

Fig. 3. Real-time optimal feedback control through inner-outer loop structure.

traditional linear or nonlinear control theory could be used to design the inner loop while the outer loop would use optimal control. To facilitate autonomy and cope with changing environments, the outer loop optimal control problem must be solved in real time. For example, in the control of mobile robots, rapid trajectory generation can be used in a feedforward mode to support the quick planning and re-planning of motion. In the case of multi-agent systems, real-time computation of optimal control is a necessity for autonomous operations to prevent collisions and/or maintain formations. In a purely feedforward mode, system trajectory generation would simply be used to generate the commands to alleviate the burden on the inner-loop requirements and/or to enhance the performance of the control system by providing the outer-loop control as well. A feedback of the outer loop supports a more efficient management of complexity, but requires real-time optimal control. A similar structure has been successfully applied by NASA in the attitude control of International Space Station (ISS). In this case, the PS optimal control methods are used in the outer loop to provide optimal reference trajectories.

The use of inner loop is not necessary to implement the optimal feedback control. Actually, the inner loop can be removed as long as the optimal control can be generated sufficiently fast. This structure is relatively simple and more appealing. The tread off is the increasing requirement on the computational speed. This type of feedback structure has been successfully applied in the magnetic attitude control of NPSAT1, an experimental satellite being build at Naval Postgraduate School. The ground test experiments demonstrate superior performance over traditional control methods 
like PID control and linear quadratic optimal control. The idea has also been tested in other applications such as the guidance of X-33-type vehicle reentry and descent problem; and path planning and obstacle avoidance of ground vehicles.

In principle, any optimal control methods that are capable of generating optimal solution in real-time can be used to construct the feedback closed-loop controller. What pseudospectral methods facilitate is the fast convergence rate which translates to a small computational delay and computational error, and the ability to handle a wide variety of nonlinear systems, which improves the robustness of the closed-loop structures and reduces the design cost. Due to these inherent properties, PS optimal control methods serves as a powerful engine and provides a unified framework for the construction of feedback optimal control. Interesting readers are referred to [37] for detailed discussion on the issues like definition of the solution, real-time requirements and error estimations, that are related to the closed-loop implementation of PS optimal control.

2) Infinite horizon problems: The methods presented in previous sections is focusing on solving finite-horizonal optimal control problems. They can actually be extended to infinite-horizonal problems as well, with some modifications. Since the infinite-horizonal problems are closely related to the optimal feedback control, we brief the techniques in this section. The details can be found in [10].

The key idea is to use domain transformation to map the semi-infinite domain to the half-open, finite interval, $[-1,1)$, and then use the appropriate PS discretization scheme able to accommodate the open right side of the time horizon. In the following, we focus on the rational mapping and the Legendre PS method. Similar ideas apply to other domain transformations and orthogonal polynomials.

For $t \in[0, \infty)$ and $\tau \in[-1,1)$ let

$$
t=\frac{1+\tau}{1-\tau} \Leftrightarrow \tau=\frac{t-1}{t+1}
$$

Using (7) and its derivative,

$$
\frac{d t}{d \tau}=\frac{2}{(1-\tau)^{2}}:=r(\tau)
$$

we can reformulate an infinite horizon problem over the finite interval $[-1,1)$. For the purpose of simplicity, we abuse notation in not distinguishing between $t(\tau)$ and $\tau$ for functional dependencies and state the transformed problem as: determine the state-control function pair $[-1,1] \ni \tau \mapsto$ $\left\{x \in \mathbb{R}^{N_{x}}, u \in \mathbb{R}^{N_{u}}\right\}$ that minimizes the cost functional,

$$
J[x(\cdot), u(\cdot)]=\int_{-1}^{1} F(x(\tau), u(\tau)) r(\tau) d \tau
$$

subject to the dynamics,

$$
\frac{d x}{d \tau}=r(\tau) f(x(\tau), u(\tau))
$$

initial conditions $x(-1)=x_{0}$ and path constraints $h(x(\tau), u(\tau)) \leq 0$. It should be emphasized that in this formulation, all functional evaluations at $\tau=1$ is equivalent to the limit of the original function as $t \rightarrow \infty$ [10].
Next, we approximate the trajectory by $N$-th order Lagrange interpolating polynomials over Legendre-GaussRadau (LGR) nodes, i.e.

$$
x(\tau) \approx x^{N}(\tau):=\sum_{k=0}^{N} x^{N}\left(\tau_{k}\right) \phi_{k}(\tau),
$$

where $\phi_{k}(\tau)$ are the Lagrange interpolating polynomials. The LGR nodes, $\tau_{k}$, are defined by the initial point, $\tau_{0}=$ -1 , and by the zeros of $L_{N}+L_{N+1}$ where $L_{N}$ is the Legendre polynomial of degree $N$. For these points which are distributed over $[-1,1)$, evaluation at the right-hand point (which for the mapped domain corresponds to $\infty$ ) is at $\tau_{N}=1-\epsilon$, where the size of $\epsilon$ depends inversely on $N$; that is, $\epsilon \rightarrow 0$ as $N \rightarrow \infty$. It is worth mentioning that the distribution of the LGR nodes on $[-1,1)$ is much denser near the -1 end than near the +1 end. This feature favors closed-loop control since only the control signal close to the initial node is implemented.

The derivative of the $i$-th state $x_{i}(\tau)$ at the LGR node $\tau_{k}$ is approximated by

$\dot{x}_{i}\left(\tau_{k}\right) \approx \dot{x}_{i}^{N}\left(\tau_{k}\right)=\sum_{j=0}^{N} D_{k j} x_{i}^{N}\left(\tau_{j}\right), i=1,2, \ldots, N_{x}$

where $D$ is a constant differentiation matrix. Finally, the integration in the cost functional $J[x(\cdot), u(\cdot)]$ is approximated by the Gauss-Radau integration rule,

$J[x(\cdot), u(\cdot)] \approx \bar{J}^{N}\left(\bar{x}^{N}, \bar{u}^{N}\right)=\sum_{k=0}^{N} F\left(\bar{x}_{k}, \bar{u}_{k}\right) r\left(\tau_{k}\right) w_{k}$

where $w_{k}$ is the LGR weights. Following these steps, the original continuous infinite-horizon optimal control is approximated by a nonlinear programming problem and thus can be solved by appropriate NLP solvers.

Based on this enabling computational technique, feedback solution to an infinite-horizonal optimal control problem can be achieved in a moving origin fashion: at every sampling instant, based on the measurement, an open loop optimal control is computed using the PS method; then this control is applied to the system over some period; the procedure is repeated over the next sampling point. This idea is explored in [32] where two feedback closed-loop algorithms were formulated. One is a free-sampling frequency approach that maximally exploits the computational power, and another based on a fixed sampling frequency that maximally exploits prediction with online optimization to reduce the effects of computational delay. Under reasonable conditions, the closed-loop system is proved to be practically stable under both computational delay and computational errors/disturbances. Detailed stability and robustness analysis can be found in [32].

3) Unified framework for control and estimation: In closed-loop implementation, estimation/observer is a necessary component. The impressive convergence property of pseudospectral methods also make them a good tool for online state estimation. In [16], a moving horizon type of numerical observer is constructed by transferring an estimation problem to a sequence of optimization problem. Consider the observer design for the following nonlinear system with 
sampled output

$$
\begin{aligned}
\dot{x} & =f(x, t) \\
y_{i} & =h\left(x\left(t_{i}\right)\right)
\end{aligned}
$$

where $y \in \mathbb{R}^{N_{y}}$ is the measurable state and $\left\{t_{i}\right\}_{i=0}^{\infty}$ is the sequence of sampling time with $\lim _{i \rightarrow \infty} t_{i}=\infty$. The estimation of the unmeasurable state can be obtained by solving the following optimization problem in real-time.

Problem E: Determine the function $z(t)$ that minimizes the cost function

$$
J[z(\cdot)]=\int_{T-\delta}^{T}\|h(z(t))-y(t)\|^{2} d t
$$

subject to the state equation $\dot{z}(t)=f(z(t), t)$ and the path constraint $r(z(t)) \leq 0$, where $T$ is the current sampling time.

This problem can then be solved by pseudospectral methods. An advantage of the observer based on pseudospectral methods is to put the control and estimation in a unified framework. Thus, the controller and observer can share the same engine — PS methods (see Fig.4). For sophisticated control systems like those in military applications, a unified framework for the control and estimation is an efficient way to manage complexity. A unified framework that is portable across heterogeneous systems is not only theoretically elegant but also reduces operational costs. For example, in the case of multi-agent systems, if each agent of a heterogeneous system has a common software, complex decisions can be quickly reprogrammed across the board. The alternative of specialized software not only increases the cost of implementation, it also increases the risk of failure as a specialized software may not be able to handle unforeseen scenarios, particularly as a result of heterogeneous interactions. In addition, a common software reduces the enormous cost associated with software verification and software management.

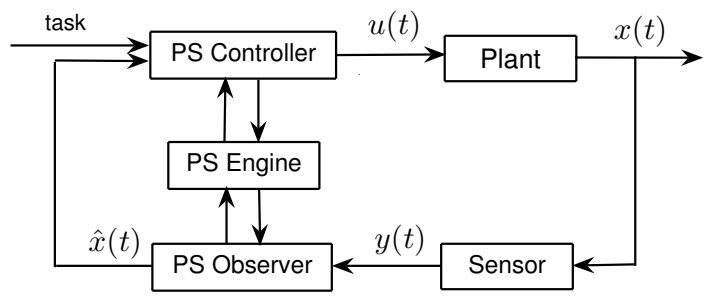

Fig. 4. Block diagram for the unified control/observer design framework by PS methods.

Many existing control methods can only be applied to a specific type of systems. A simple change in the control plant, for instance, adding a constraint, can result in a redesign of the entire feedback control law. The fact that pseudospectral methods are capable of solving both control and estimation problems for a wide variety of nonlinear systems makes them an attractive tool to build an unified framework for closed-loop control purpose. This enabling technology also facilitates a cost efficient output feedback design for nonlinear systems. The idea is illustrates in the following diagram. For the details on building a unified framework by PS methods, the readers are referred to [17].

\section{F. Conclusion}

In this section, we provide a brief overview of pseudospectral optimal control methods which server as a powerful tool to tackle complicated nonlinear control problems. Several successfully applications in various military and industrial applications are addressed in the next few sessions. The solid theoretical foundation and practical advantages make PS methods an attractive all-round optimal control algorithm for complicated nonlinear systems.

\section{First Ever Zero-Propellant ${ }^{\mathrm{TM}}$ MANEuver OF Space Station Using Pseudospectral Optimal Control Based Guidance - Nazareth S. BEDROSSIAN AND SAGAR BHATT}

This section describes the application of pseudospectral optimal control to develop a new attitude control concept for spacecraft large rotational state transitions without using propellant, Zero-Prop Maneuver $(\mathrm{ZPM})^{\mathrm{TM}}$. It also presents results for the historic ZPM flight demonstrations on November 5, 2006 and March 3, 2007 when the International Space Station (ISS) was rotated 90 degrees in 2 hours and 180 degrees in about 3 hours respectively. The propellant savings for both maneuvers are estimated at $150 \mathrm{lbs}$ with an estimated value of $\$ 1,500,000$.

With the ZPM, non-propulsive rotational state (attitude, rate, momentum) transition for spacecraft controlled by momentum storage devices can be accomplished. A rotational state transition can be a maneuver between prescribed states and/or an attitude maneuver used to desaturate the momentum actuators. For the ISS, the benefits of a ZPM include reduced lifetime propellant use, and reduced constraints on solar array operations due to loads, erosion and contamination from thrusters. It did not require ISS flight software modifications since it is a set of attitude and rate commands tailored to the specific attitude control architecture. More importantly, ZPM provides the only means by which to rotate the ISS in case thruster control capability is lost.

Prior to development of the ZPM method, ISS large angle attitude maneuvers were performed using thrusters. Though the ISS is equipped with Control Moment Gyroscopes (CMGs) that are used for attitude control, the CMGs have limited torque and momentum capability. Maneuvers are typically between torque equilibrium attitudes i.e., attitudes that can be held long term by the CMGs without momentum saturation. For short term attitude hold and maneuvers, a PID attitude hold controller with an eigenaxis maneuver logic is used. Commanding a large angle maneuver with the flight software would cause the CMGs to rapidly reach their operational limits requiring thrusters for desaturation. Due to CMG lifetime issues, momentum desaturation using thrusters is currently prohibited.

The ZPM concept is based on using a commanded attitude trajectory to accomplish the desired rotational state 
transition. The trajectory is shaped in a manner that takes advantage of the nonlinear system dynamics to reduce or eliminate the "cost" of the maneuver. For example, an eigenaxis maneuver is kinematically the shortest path between two orientations. For the attitude controller system to follow the eigenaxis, the nonlinear system dynamics must be overcome, thereby increasing the "cost" of the maneuver. By considering a kinematically longer path and increasing the time to perform the maneuver, path dependence of system dynamics can be exploited to lower the "cost". This allows spacecraft actuated by momentum storage devices, such as the ISS, to perform large angle attitude maneuvers nonpropulsively.

ZPM is a direct result of advances in computational capabilities and development of commercially available software packages that can solve optimal control problems for complex nonlinear dynamical systems. In recent years, advances in PseudoSpectral (PS) methods have allowed for the efficient and rapid solution of optimal control problems governed by arbitrary nonlinear dynamical systems. PS methods differ from other techniques in several different ways. Because they are based on discretizing the problem by way of Lagrange interpolating polynomials over quadrature nodes, they offer spectral accuracy (i.e., a faster convergence rate than any given polynomial rate) which provides the efficiency required for flight applications. In contrast, prior methods typically offer only order four convergence. Furthermore, PS methods offer a simple way to check the optimality of the solution by way of the Covector Mapping Principle. This concept is particularly important in solving a complex problem like the ZPM because it facilitates quick and efficient ways to validate the feasibility and optimality of the solution.

A PS approach was used to solve the ZPM optimal control problem using 2003a version of the software package DIDO [34], which implements the Legendre PS method in an object-oriented framework under MATLAB . DIDO uses a spectral algorithm in conjunction with SNOPT [11], an active-set sequential quadratic programming solver, to generate fast ZPM solutions.

To implement the ZPM, the ground-developed trajectory is converted into a Greenwich Mean Time (GMT) time-tagged command pair sequence for uplink to the ISS Command and Control computer (C\&C MDM) prior to the maneuver execution time. As the C\&C MDM command buffer is limited to 200 slots, the non-propulsive maneuver is allocated 160 slots. This limits the ZPM to 80 quaternion commands and 80 maneuver rate commands. For the 90deg maneuver the commands were updated every $90 \mathrm{sec}$, while for the $180 \mathrm{deg}$ maneuver the commands were updated every $125 \mathrm{sec}$.

Telemetry from each ISS ZPM will be presented as well as animation of the maneuvers. Flight data for the ZPM attitude trajectories and the associated CMG momentum for the 90degree and 180degree maneuvers are shown in the following figures. It is seen that for 90degree maneuver the peak momentum only reached $70 \%$ while for the 180degree maneuver it was $76 \%$.

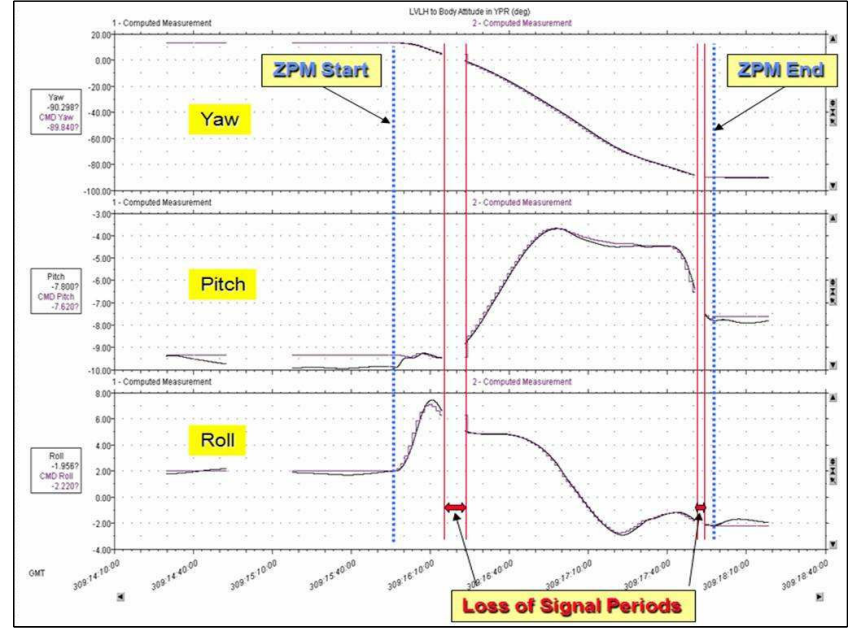

Fig. 5. 90degree ZPM ISS telemetry for commanded and actual attitude.

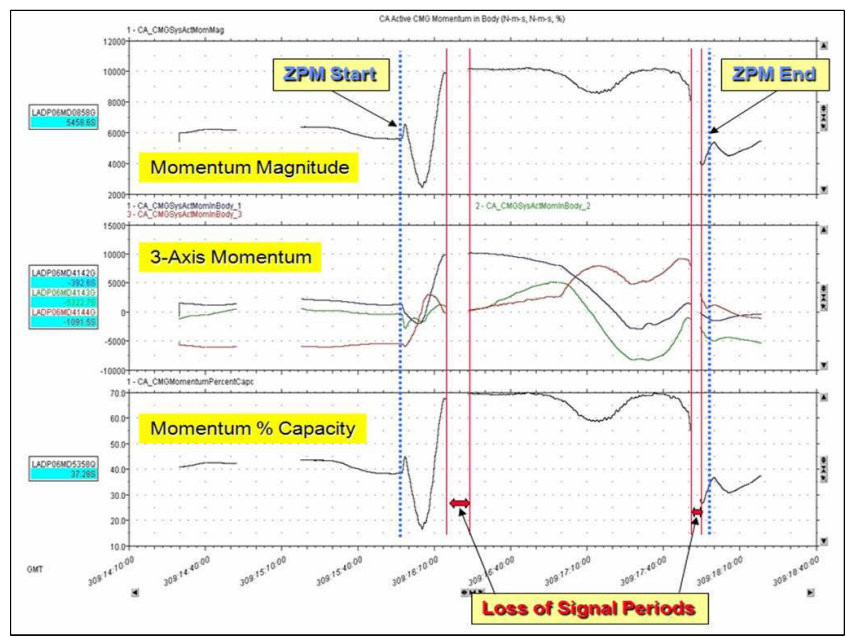

Fig. 6. 90degree ZPM ISS telemetry for CMG momentum.

\section{A Unified View ON Discrete Optimality CONDitions For PSEUdospeCtRal Methods - FARIBA FAHROO}

In recent years, a vast number of optimal control problems have been solved by pseudospectral methods; examples include the design and control of formations, formation reconfiguration problems, cycler trajectory design using solar sails, sample return missions, ascent and entry problems, asteroid sample return missions, attitude control problems, benchmark trajectory optimization problems and many others. As a result of its versatility, pseudospectral methods will also be available in the next generation of NASA's OTIS software package [29]. Details of these plans are described in http://trajectory.grc.nasa.gov/projects/lowthrust.shtml. The essential idea of making pseudospectral (PS) methods available through OTIS is that users who are familiar with the OTIS interface can now make avail of PS methods simply by setting a few options.

The most widely used pseudospectral method is the Leg- 


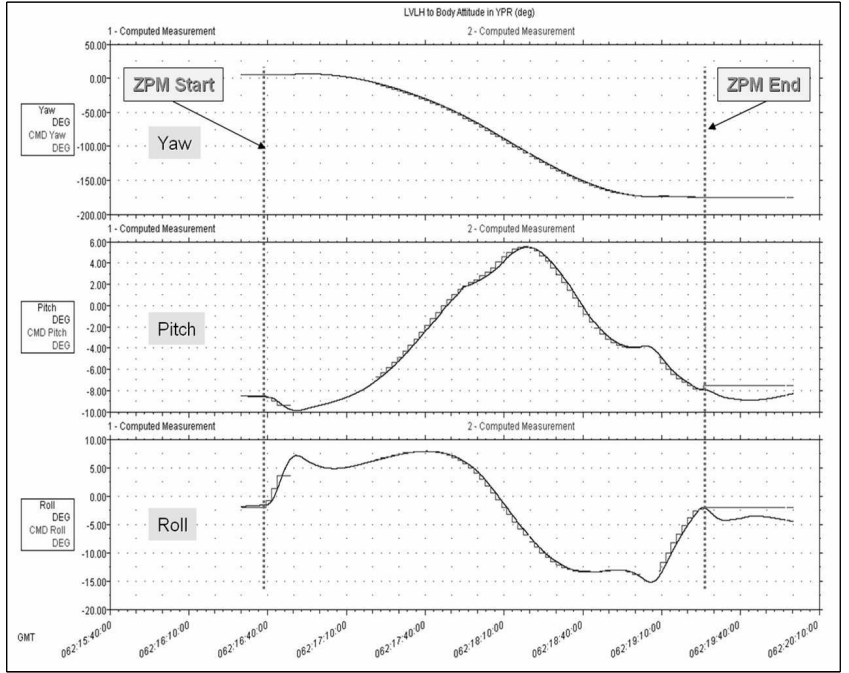

Fig. 7. 180degree ZPM ISS telemetry for commanded and actual attitude.

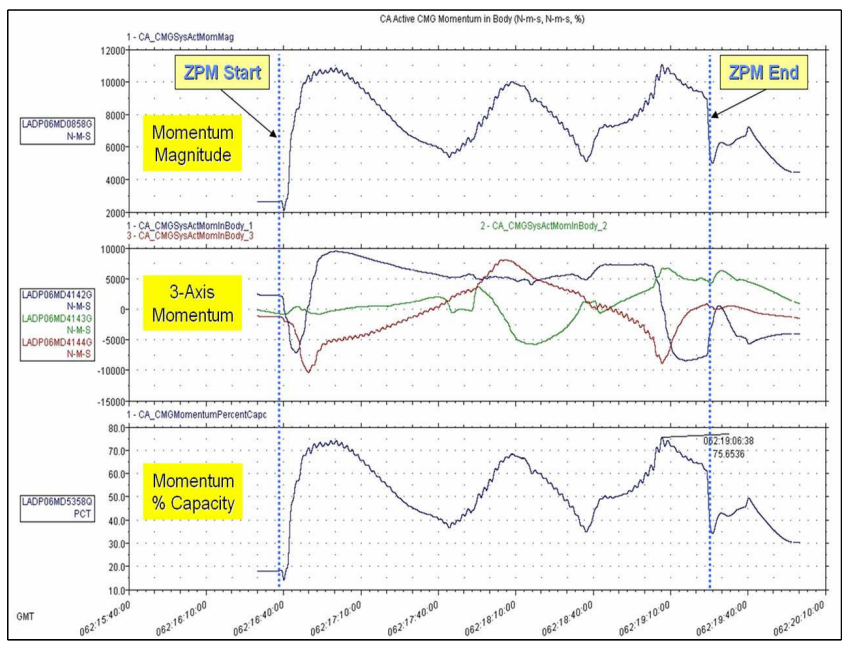

Fig. 8. 180degree ZPM ISS telemetry for CMG momentum.

endre PS method. This is simply because the Legendre PS method is known to satisfy the Covector Mapping Principle (CMP) by way of an explicit mapping condition given by a Covector Mapping Theorem. The proof of this theorem utilizes specific quadrature formulas that are valid only for the Legendre PS method. Thus, a natural question that remains unanswered is whether other PS methods (such as the Chebyshev PS method or the recently introduced Legendre-Radau method) satisfy the CMP. An apparently simply way to investigate this issue is to derive an explicit covector map in a manner similar to the Legendre-GaussLobatto (LGL) approach. While conceptually simple, this task is not altogether straightforward as a key lemma related to an integration-by-parts formula is not readily available for non-LGL methods. This crucial formula identifies the correct discrete inner-product space that is necessary for the construction of the discrete 1-form that defines the sequences of discrete Lagrangians that converge to continuous- time Lagrangians. We identify this inner-product space by investigating the problem at the level of first principles. That is, we first construct PS approximations to the optimal control problem over an arbitrary grid. In the same spirit, we construct PS approximations to the optimality conditions of the continuous-time problem by approximating the equations resulting from an application of the Minimum Principle. As has been noted before, the Karush-Kuhn-Tucker (KKT) conditions for the discrete problem does not, in general, resemble a discretization of the continuous-time conditions. While this does not mean that a covector map does not exist, the crucial problem here is to investigate the existence of such a map. In this paper, we derive an explicit transformation between the KKT multipliers and the PS discretization of the necessary conditions. This transformation requires the satisfaction of additional or closure conditions. When the LGL points are substituted in this new transformation, we recover the original mapping theorem. For non-LGL points, we develop a family of transformations and demonstrate numerically, by solving an orbit transfer problem, that a satisfaction of the CMP does not imply convergence. In this context, we not only generalize and extend our prior results, we also show that, contrary to popular belief, the existence of a covector transformation does not imply convergence although convergence implies a satisfaction of the CMP. Complete mathematical details including numerical examples will be provided.

\section{NPSAT1 Time-Optimal Slew Maneuvering: Ground Test Results - Pooya Sekhavat}

Time-optimal steering of a spacecraft can substantially improve its performance through rapid reorientations. However, due to the lack of an effective method to solve the time-optimal slew problem in its general form, the common practice thus far has been limited to the eigenaxis maneuvers. As a result of the recent breakthroughs in pseudospectral (PS) control, it is now possible to substantially enhance the performance of spacecraft slew maneuvers at reduced cost through closed-loop time-optimal feedback control. This revolution in feedback control is obtained by recognizing that closed-loop does not necessarily imply closed-form solutions. Given that pseudospectral methods can demonstrably generate open-loop optimal solutions in fractions of a second to a few seconds, the premise of this work is to show that the closed-loop optimal feedback control can be obtained by real-time computation of open-loop optimal solutions. The possible control discontinuities in the open-loop segments are addressed by defining a solution over the sample segment in the standard Carathéodory sense, and then glue the pieces in the same manner as in the $\pi$-trajectory. We distinguish this concept as a Carathéodory- $\pi$ trajectory, i.e., when open-loop controls are generated fast enough, closed loop control can be achieved via generating Carathéodory- $\pi$ solutions [37]. The resulting clock-based Carathéodory- $\pi$ feedback control scheme has further a built-in safety factor (compared to traditional feedback controls) in the following sense: if the feedback signal were cut off, the last open-loop optimal 
control trajectory remains as the plant input for the rest of the maneuver. Another desirable feature inherent in such control algorithm is the fact that it is "gain-free" and does not require the user to select or tune any controller gain; rather, "designer functions" would be automatically generated at the fundamental computational level.

This section presents the results of applying the above explained pseudospectral feedback control to the slew maneuvering of NPSAT1, a prolate non-spinning body designed and built at the Naval Postgraduate School and scheduled to launch in 2009. It employs magnetic sensing and actuation for attitude control which leads to a highly nonlinear and time-varying dynamic system. Because the pseudospectral feedback control exploits the full maneuverability envelope of the complete nonlinear system, it easily outperforms the traditional gain-based feedback control laws even in the presence of various uncertainties and exogenous disturbances [42], [43].

Figs 9 and 10 show the successful performance of the method for a 135 degree rest-to-rest $\mathrm{x}$-axis maneuver with and without an exaggerated exogenous disturbance torque.

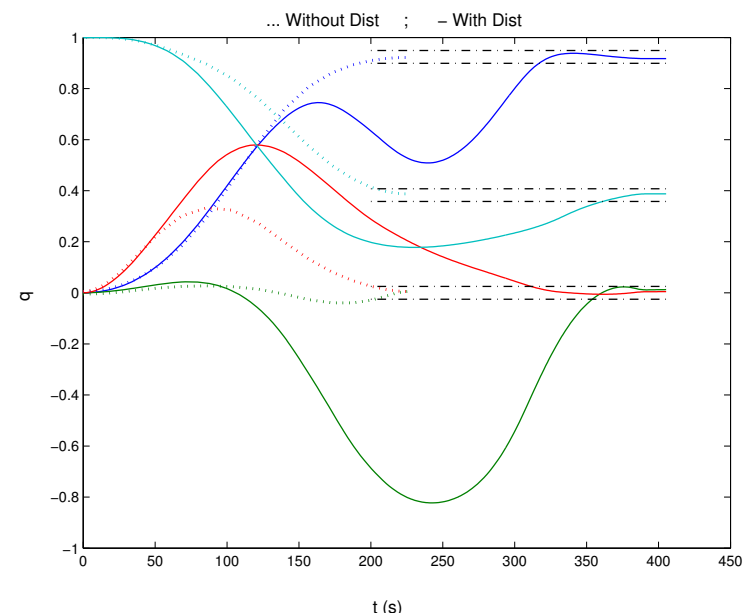

Fig. 9. NPSAT1 closed-loop time optimal position response.

Specially when traditional control algorithms lead to false infeasibilities of certain performance metrics such as the ability of the spacecraft to perform a horizon-to-horizon look, the PS-based Carathéodory- $\pi$ feedback control scheme is shown to be able to unveil the true system capabilities in conducting the same mission (see Ref [42]). It is also shown that the proposed PS-based Carathéodory- $\pi$ feedback control is capable of exploiting the benefits of potentially favorable exogenous disturbances rather than rejecting them as is commonly done with traditional feedback controllers.

Although necessary, computer modeling and simulated demonstrations alone do not provide a complete practical assessment on the maturity, capabilities, and benefits of such a revolutionary technology. It is imperative to develop prototype tests reflecting the capabilities of this new notion of feedback optimal control through experiments. In that regard,

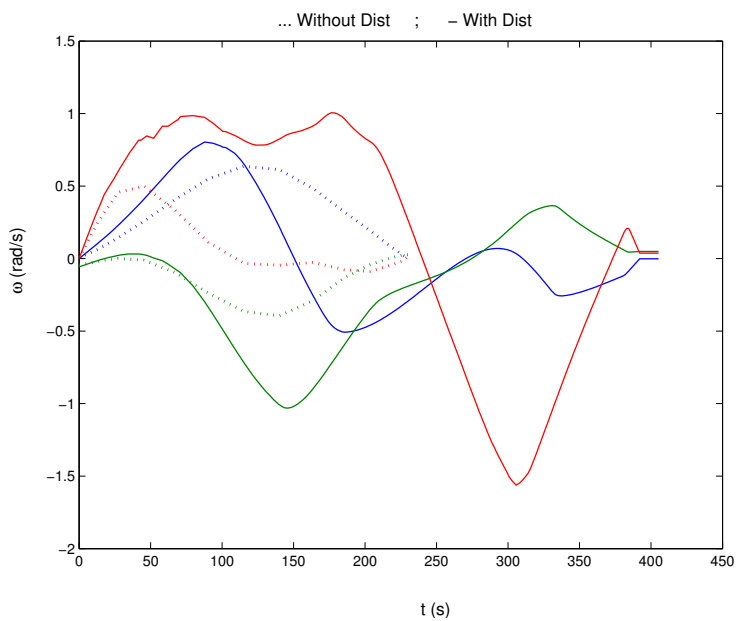

Fig. 10. NPSAT1 closed-loop time optimal angular velocity response.

the PS-based Carathéodory- $\pi$ feedback control algorithm has been implemented on the NPSAT1 laboratory-scale test-bed.

Fig 11 shows the airbearing table used for NPSAT1 ground tests. Similar to NPSAT1, the airbearing platform employs a three-axis magnetometer for magnetic sensing and three magnetic torque rods for actuation. Interaction between the three magnetic dipole moments generated by the torque rods and the Earth's magnetic field produces the control torque. The optimal control problem is the rest-torest maneuvering of the spacecraft to the desired attitude in minimum time. Thus, the cost function is defined as the maneuver time from the initial to the target attitude. The open-loop optimal control trajectories are obtained using the PS-based software package, DIDO. Computed controls are then transmitted to the onboard single board computer (SBC) through a wireless link and stored on it as series of timecontrol pairs. The pairs are then sent by the SBC to the torque rod microcomputer for implementation until the next update of the open-loop trajectory. Having a magnetometer as the only available sensor onboard rules out any possibility of direct position and/or angular rate measurement. The attitude and angular rates should, therefore, be estimated based on the magnetometer readings. The instantaneous state values required for feedback generation throughout the experiments are derived by incorporating an Unscented Kalman Filter into the control program. Another main feature of the ground motion is that, unlike NPSAT1 in orbit, the table center of mass does not coincide with the center of rotation. This generates a gravity torque that can be considerably larger than the maximum actuation torque that can be generated by torque rods. Therefore, the gravity torque is not treated as disturbance and is included in the NPSAT1 ground model that DIDO uses for control generation.

As a result of the nature of the in-house fabrication and assembly of the test-bed, it is not possible to accurately measure all the system parameters. Such parameter uncertainty is treated as a disturbance for the feedback control. The results shown in Fig 12 show the performance of the PS- 


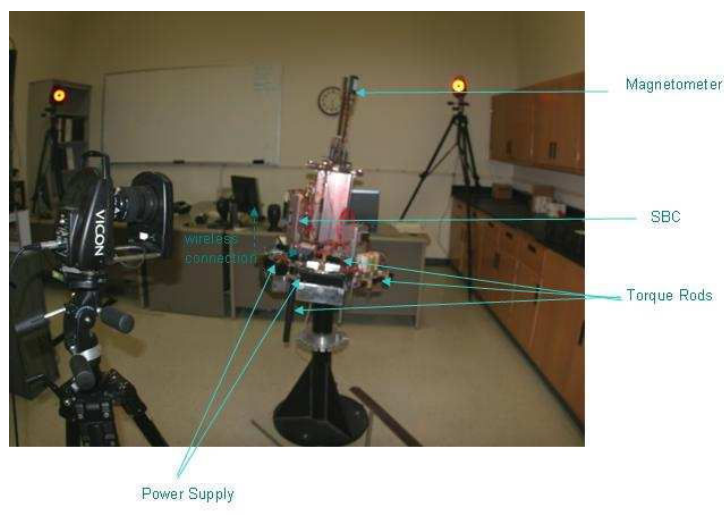

Fig. 11. NPSAT1 ground test-bed.

based closed-loop control during a 135 degree slew maneuver in the presence of parameter uncertainties in moments of inertia and the distance between the center of mass and center of rotation. It is seen that the PS-based Carathéodory$\pi$ feedback control steers the spacecraft to the target attitude successfully.

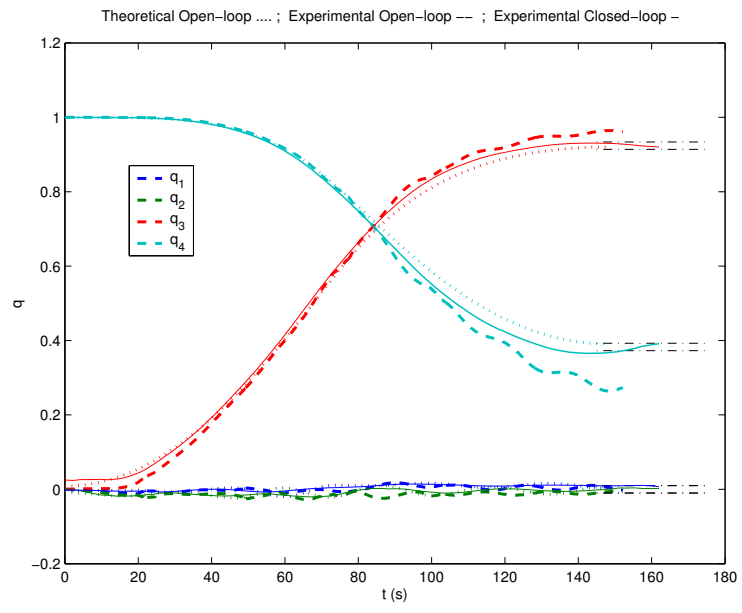

Fig. 12. NPSAT1 ground test results.

Further ground tests has been conducted to evaluate the experimental performance of the control algorithm in reaction to exogenous torques. The results show successful slew maneuvers even when the system was unexpectedly disturbed via an external "kick" during the motion.

\section{Pseudospectral Motion Planning For Autonomous Obstacle Avoidance - Qi Gong And$$
\text { L. R. LEWIS }
$$

Military applications of unmanned and autonomous vehicles have drawn considerable interest and recognition in recent years. Most notable is the Unmanned Aerial Vehicles (UAV), but considerable advances are making the incorporation of unmanned Ground Vehicles (UGV) and Sea Surface Vehicles possible. These systems remove humans from jobs that would be otherwise extremely dangerous. A common task in these military robotic applications is the autonomous trajectory planning. It allows the vehicle to travel from one location to another safely, within the limits of its electrical and mechanical capabilities, and in some situations, in an optimal manner with respect to use of fuel, expenditure of time, or distance travelled.

The various applications of trajectory planning led to the evolution of differing techniques to solve their problems. Aeronautical and space applications drove the development of nonlinear optimal control techniques. In these situations, global problem knowledge is assumed, vehicle motion is precisely understood, few physical constraints obstruct the vehicular motion, and trajectory optimization is key to design and mission success. Robotics applications propelled a differing solution, but it was a solution better adapted to fit the inherent situational complexities including: limited problem knowledge, noisy sensors, uncertain dynamical characteristics, intricate obstacle-rich environments, limited computational power, and the necessity to generate feasible solutions. Despite the general similarity between problems, these two fields of application experienced little crosspollinization. Aerospace applications desired optimality and robotics applications desired simplicity and an ability to handle uncertainty.

Traditionally, optimization based trajectory planning created solutions in a none real-time fashion due to the enormous computational burden. It limits the application of optimal control methods in robotics-based application. However, recent advances in computational nonlinear optimal control and exponentially growing computing power are changing that paradigm and research is showing the applicability of optimal control techniques in real-time guidance and control. Using these advanced techniques, even for complicated applications solutions can now be found within seconds or less, and the improvements in computational speed increase their ability to handle uncertain environment. The improvements in computational optimal control methods present a strong argument for their application in online planners and with robotics problems. Given the progress made in applying optimal control methods to online aerospace applications and being aware of the ability to eliminate the associated computational burden, this paper focuses on the application of these techniques to a new breed of problems, sharing attributes with both aerospace and robotics applications. This problem is that of the unmanned vehicle, characterized by a lack of global knowledge, complex obstacle-rich environments, and a need for feasible solutions in the face of uncertainty. On the other hand, these systems typically incorporate sophisticated sensors and ample computational power.

The research presented here evaluates and validates the concept of trajectory planning for unmanned vehicles with optimal control methods. The primary thrust of this work is the optimization of kinematic trajectories within varying, complex environments. The computational method we adopt is a pseudospectral (PS) method due to the superior convergence property that is crucial for real-time applications. 
1) Vehicle Model: In this research we limit our focus on the problem of generating minimum-time trajectories for autonomous vehicles. Two types of vehicles are considered. The first is a commonly used four-wheeled car with rearwheel drive and front-wheel steering. This system is wellstudied, and the nonholonomic-nature of the constraints adds kinematic complexity. A simple, kinematic model [4] of the car is shown in (8).

$$
\begin{aligned}
\dot{x} & =v \cos (\theta) \\
\dot{y} & =v \sin (\theta) \\
\dot{\theta} & =\frac{v}{L} \tan (\phi)
\end{aligned}
$$

The $x-y$ location of the car represents the current position of the center point of the rear axle. The car's orientation angle is measured with respect to the horizontal axis and is presented as the state variable $\theta$. The steering angle, $\phi$, is measured with respect to the car's heading, or velocity vector, and the variable ' $L$ ' measures the distance between the front and rear axles. The speed $v$ and steering angle $\phi$ are the control variables satisfying the following constraints.

$$
\begin{aligned}
v_{\min } & \leq v \leq v_{\max } \\
\phi_{\min } & \leq \phi \leq \phi_{\max }
\end{aligned}
$$

The steering angle must be between $\pm 90^{\circ}$ for computational and for practical purposes it is typically much less than that. Note that unlike the Reeds-Shepp or Dubin's cars the velocity is allowed to change continuously between the extremes similarly to the capability of a real car. The second vehicle used is a simple UAV [46] modeled by

$$
\begin{aligned}
\dot{x} & =v \cos (\gamma) \cos (\xi) \\
\dot{y} & =v \cos (\gamma) \sin (\xi) \\
\dot{z} & =v \sin (\gamma)
\end{aligned}
$$

The state variables represent the three spatial degrees of freedom. The three control variables are the vehicle velocity, $v$, the flight path angle, $\gamma$, and the heading angle, $\xi$. The flight path angle is measured from the local horizontal to the velocity vector, and the heading angle is measured from a reference heading - e.g. due North - to the velocity vector.

2) Obstacle Representation: For simplicity and computational efficiency it is desirable to represent the path constraints as continuous, differentiable algebraic functions. With this framework in mind, the p-norm was used to create generic shapes including: diamonds, circles and ellipses, and squares and rectangles. Define the p-norm as

$$
\|(x, y)\|_{p} \triangleq\left(|x|^{p}+|y|^{p}\right)^{\frac{1}{p}}, p=1,2, \cdots .
$$

Then the obstacle can be represented by the following path constraints:

$$
h(x, y)=\left\|\left(\frac{x-x_{c}}{a}, \frac{y-y_{c}}{b}\right)\right\|_{p}-c,
$$

where constants $a$ and $b$ are used to scale the $x-y$ axes, and constant $c$ is the radius. Any point outside the obstacles yields a path constraint value greater than zero. Fig.13 demonstrates how the p-norm can be manipulated to create these diamonds, circles and squares obstacle shapes; the ellipse and rectangle are simply extensions of the circle and square respectively where the distance along the $\mathrm{x}$ and $\mathrm{y}$ axes are dissimilar. The technique can be extended to cover more
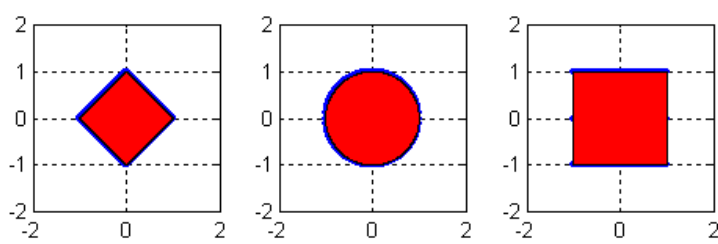

Fig. 13. Unit $p$-norms for $p=1,2,100$ respectively.

general polygonal obstacle shapes [26].

3) Results: In the $\mathrm{X}-\mathrm{Y}$ coordinate frame, the vehicle is intended to travel from the location $(0,0)$ and oriented down the $\mathrm{X}$-axis to the location $(10,10)$. The velocity is between $[-0.2,0.2]$ and the steering angle is within $\left[-35^{\circ}, 35^{\circ}\right]$. The optimal vehicle state trajectory, as created using software package DIDO [34], is shown in Fig.14. There is a noticeable distance between the vehicle trajectory and the side of the obstacle; the reason for this separation is in accounting for the size of the UGV. The UGV trajectory is straight unless

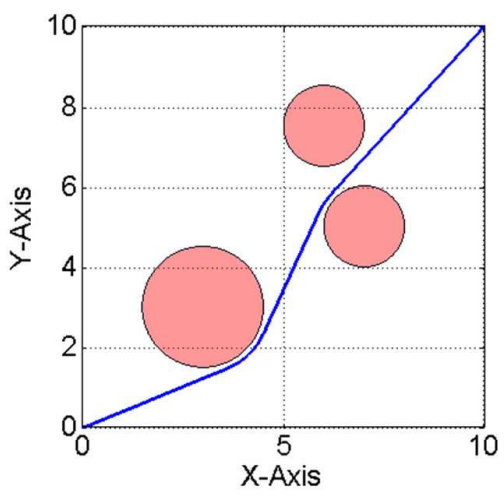

Fig. 14. Time-optimal UGV trajectory through three circular obstacles.

it is maneuvering to avoid an obstacle, thus displaying the optimal nature of the result. The optimal control trajectory that generated this state path is shown in Fig.15. The optimality of the calculated trajectories can be verified by way of the necessary conditions [26].

Next we test the ability to find a solution in the event of UGV damage. Fig.16 shows the optimal control trajectory when the vehicle must turn left, i.e. the minimum and maximum steering angle values are positive. In this situation, the solution is not initially intuitive or well understood. The time-optimal result requires two types of motion. The first type combines forward motion of the vehicle with a minimum steering angle; this results in a maximum turning radius. The second type of motion combines the backward motion of the vehicle with a maximum steering angle; this results in a minimum turning radius. In total, the UGV moves forward with a minimum turning radius and then 

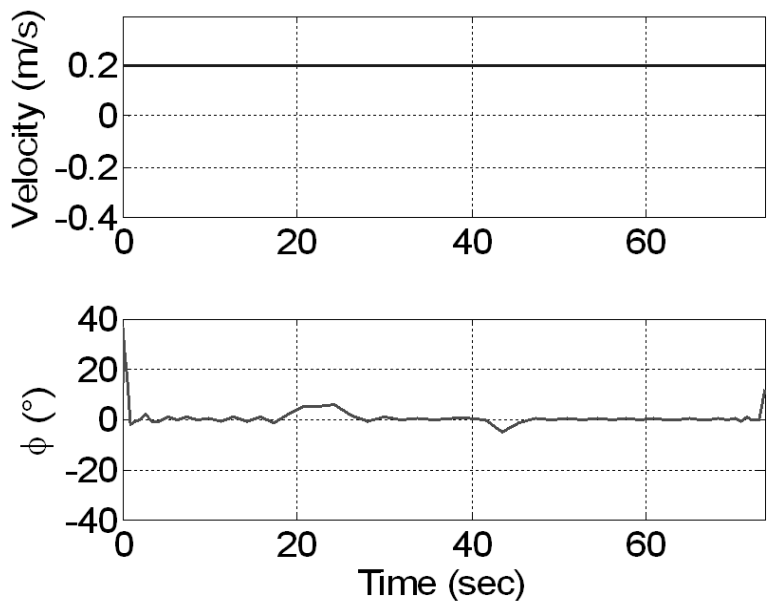

Fig. 15. Time-optimal UGV controls through three circular obstacles.

backward with the maximum turning radius to align for the next forward motion. This type of motion is used in a repetitive fashion in Fig.16 to successfully navigate the obstacles.

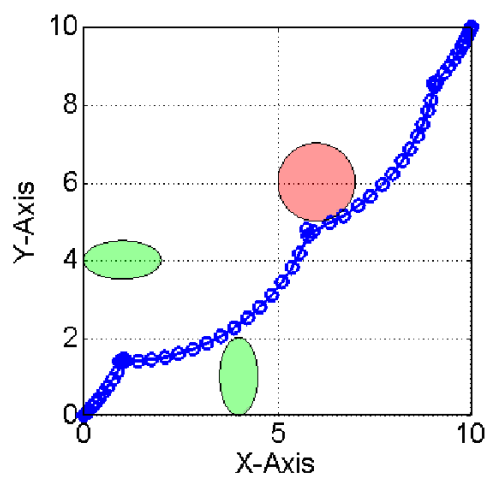

Fig. 16. Time-optimal maneuver with a control failure.

The minimum time trajectory planning for UAV under urban environment is shown in Fig.17. Due to its threedimensional nature, the problem is shown from multiple viewing perspectives in Fig.17 and the primary purpose is to illustrate the fact that the trajectory does not violate any building constraints. The feasibility of the optimal control to the nonlinear dynamic is shown in Fig.18.

These results further illustrate the portability and applicability of optimal control techniques. In general, this problem is more complex, but it is solved in an identical manner to the UGV problem. For this reason, it can be deduced that the same technique is portable to any vehicle whether it is submerged, sea surface, ground, or air, and the transfer from one vehicle to the next requires no change in approach.

\section{Pseudospectral-BAsed Optimal Trajectory GENERATION FOR AUTONOMOUS REENTRY AND Descent of Reusable LAUnCH Vehicles - MAJOR KEVIN BOLLINO, USAF}

Perhaps one of the most complex aerospace problems facing engineers over the past few decades has been reentry

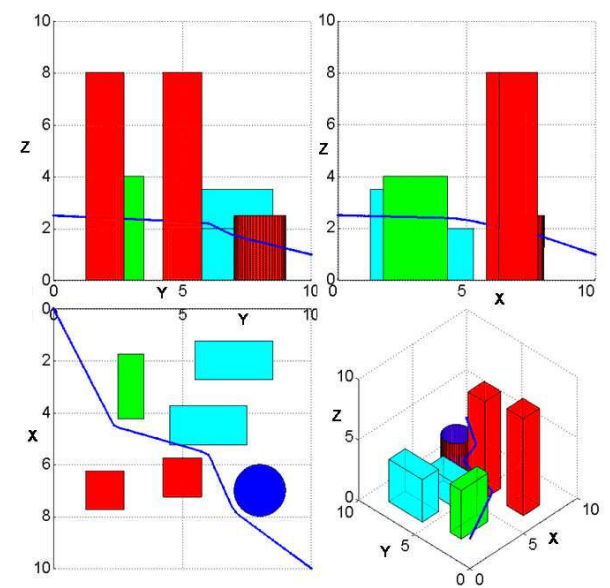

Fig. 17. Time-optimal UAV trajectory through a city environment.

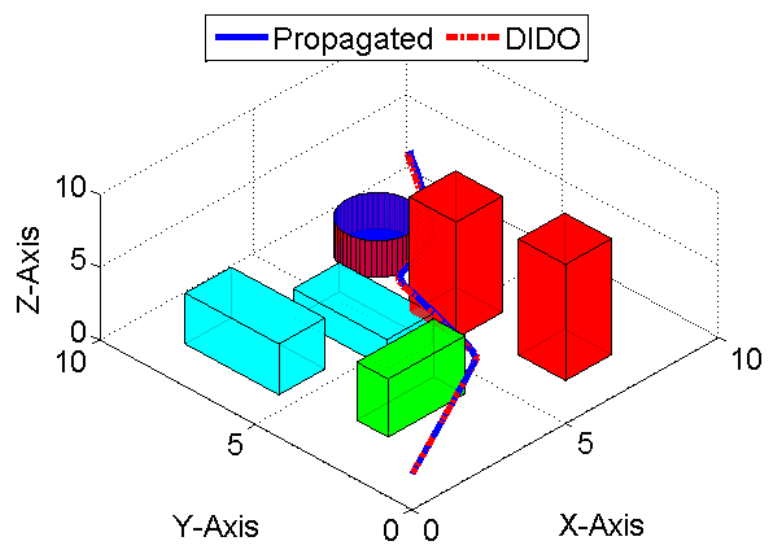

Fig. 18. Feasibility test of time-optimal UAV trajectory.

and descent of hypersonic, unpowered Reusable Launch Vehicles (RLV).

In addition to the highly nonlinear vehicle dynamics, the environment is rapidly changing and often unpredictable as an RLV descends through various layers of the atmosphere. However, amid an era of revolutionary developments in computational power and numerical methods, such previously considered "hard" problems are now, not only readily solvable, but solvable in an optimal fashion. The pseudospectral (PS) method, combined with the principles of nonlinear optimal control theory, is one such method that has demonstrated to be an effective tool for reentry applications.

The presented research focuses on automatically generating an optimal flight path and control sequence to guide an RLV from the upper atmosphere to the neighborhood of a designated runway.

With an escalating demand for onboard flight trajectory determination, guidance command generation, and robust/optimal control, the proposed techniques provide a powerful, yet simple and safe approach that addresses the emerging needs of next generation autonomous, unmanned flight vehicles. 


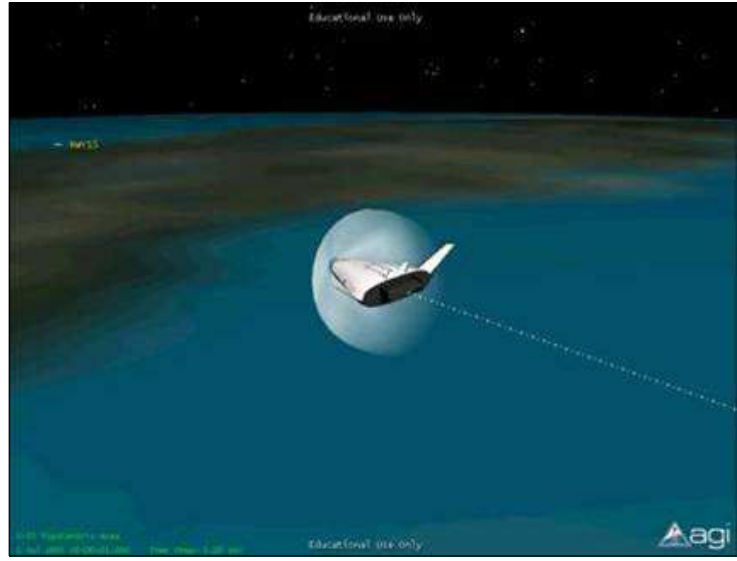

Fig. 19. Simulation of RLV Reentry.

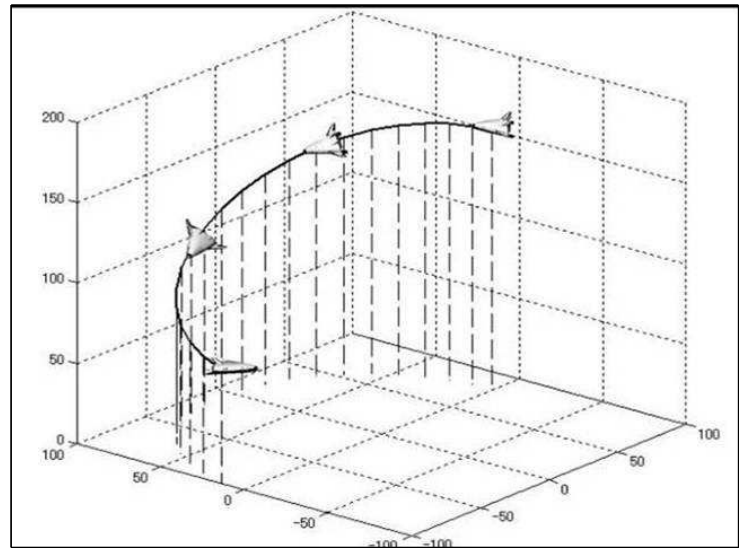

Fig. 20. Illustration of Reentry and Descent.

First, open-loop trajectory generation is demonstrated with specific scenarios including range maximization, footprint generation, intelligent maneuvers, and runway retargeting based on automatically generated Final Approach Corridors (FAC) as depicted in Fig.21.

The FAC-generation logic proves to be an efficient and effective means of rapidly defining target conditions based on the desired runway's geometry and orientation. These conditions are mapped to the optimization problem and assume that final flight path attitude and airspeed are consistent with required landing constraints. As such, the overall goal is to minimize the miss distance to the center of the generated FAC-target represented by the performance index,

$$
\begin{aligned}
J[x(\cdot), u(\cdot), t]= & \left(r_{F A C}-r_{f}\right)^{2}+\left(\lambda_{F A C}-\lambda_{f}\right)^{2} \\
& +\left(\mu_{F A C}-\mu_{f}\right)^{2}
\end{aligned}
$$

where the terms $(r, \lambda, \mu)$ represent the RLV's altitude, latitude, and longitude, respectively.

Then, the notion of a Carathéodory- $\pi$ solution is used to design, develop and implement a PS-feedback guidance algorithm capable of successfully guiding an X-33-type vehicle in the presence of large disturbances, g-load and heating-rate constraints to a designated landing site.

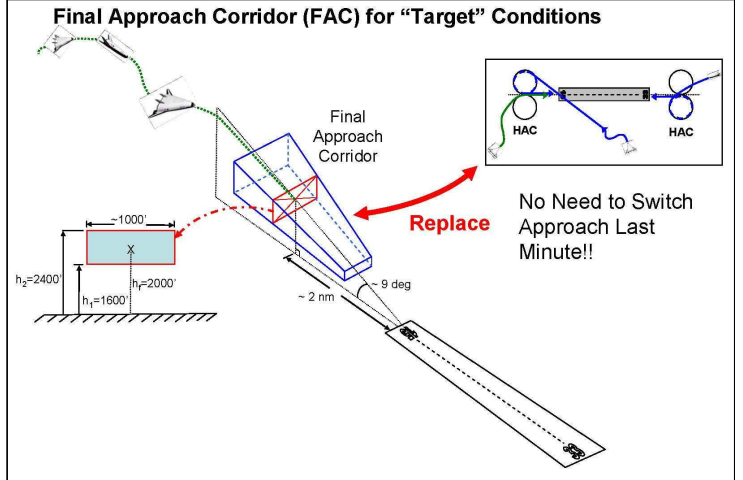

Fig. 21. Final Approach Corridor for "Target" conditions.

An added benefit of the proposed approach is its portability for use in similar flight vehicle applications. For example, on a smaller scale, the same techniques can be applied to Unmanned Aerial Vehicle (UAV) flight path-planning and control. With the use of similar flight equations of motion and updated vehicle parameters with corresponding aerodynamic models, the primary difference, independent of flight performance, would be the applied constraint sets. The path constraints could also include "no-fly zones" suitable for both military applications as well as commercial aviation applications.

Overall, the PS approach of rapidly generating optimal trajectories for challenging RLV problems proves viable for future use.

\section{REFERENCES}

[1] J. T. Betts, N. Biehn and S. L. Campbell, Convergence of nonconvergent IRK discretizations of optimal control problems with state inequality constraints, SIAM Journal Scientific Computation Vol. 23, No. 6, pp. 1981-2007, 2002.

[2] J. T. Betts, Practical Methods for Optimal Control Using Nonlinear Programming, SIAM, Philadelphia, PA, 2001.

[3] C. Canuto, M. Y. Hussaini, A. Quarteroni and T. A. Zang, Spectral Method in Fluid Dynamics, New York: Springer-Verlag, 1988.

[4] H. Choset, et al., Principles of robot motion; theory, algorithms, and implementation, The MIT Press, 2005.

[5] J. Cullum, Finite-dimensional approximations of state constrainted continuous optimal problems, SIAM J. Control, Vol. 10, pp. 649-670, 1972.

[6] A. L. Dontchev, Discrete approximations in optimal control, Nonsmooth Analysis and Geometric Methods in Deterministic Optimal Control, IMA Vol. Math. Appl., 78, Springer, New York, 1996, pp. 59-81. MR 97h:49043.

[7] A. L. Dontchev and W. W. Hager, The Euler approximation in state constrained optimal control, Mathematics of Computation, Vol. 70, pp. 173-203, 2001.

[8] G. Elnagar, M. A. Kazemi and M. Razzaghi, The pseudospectral Legendre method for discretizing optimal control problems, IEEE Trans. Automat. Contr. Vol. 40, pp. 1793-1796, 1995.

[9] F. Fahroo and I. M. Ross, Costate estimation by a Legendre pseudospectral method, AIAA Journal of Guidance, Control and Dynamics, Vol. 24, No. 2, pp. 270-277, 2001.

[10] F. Fahroo and I. M. Ross, Radau pseudospectral methods for infinitehorizon nonlinear optimal control problems, Proc. AIAA Guid., Nav. and Control Conf., San Franscro, CA, 2005.

[11] P. E. Gill, W. Murray and M. A. Saunders, SNOPT: an SQP algorithm for large-scale constrained optimization, SIAM J. of Opt., Vol. 12, No. 4, pp. 979-1006, 2002. 
[12] Q. Gong, W. Kang and I. M. Ross, A pseudospectral method for the optimal control of constrained feedback linearizable systems, IEEE Trans. Auto. Cont., Vol. 51, No. 7, July 2006, pp. 1115-1129.

[13] Q. Gong, I. M. Ross, W. Kang and F. Fahroo, Connections between the covector mapping theorem and convergence of pseudospectral methods for optimal control, to appear in Computational Optimization and Applications, 2008.

[14] Q. Gong, I. M. Ross, W. Kang and F. Fahroo, On the pseudospectral covector mapping theorem for nonlinear optimal control, 45th IEEE Conf. on Decision and Control, pp. 2679-2686, San Diago, Dec. 2006.

[15] Q. Gong and I. M. Ross, Autonomous pseudospectral knotting methods, 16th AAS/AIAA Space Flight Mechanics Conference, AAS 06151, Tampa, Florida, Jan., 2006.

[16] Q. Gong, W. Kang and I. M. Ross, A pseudospectral observer design method for nonlinear systems, Discrete and Continuous Dynamical Systems - Series B, Vol 8, No 3, pp. 589-611, 2007.

[17] Q. Gong, I. M. Ross and W. Kang, A unified pseudospectral framework for nonlinear controller and observer design, American Control Conference, pp. 1943-1949, New York City, NY, July, 2007.

[18] W. W. Hager, Runge-Kutta methods in optimal control and the transformed adjoint system, Numerische Mathematik, Vol. 87, pp. 247282, 2000.

[19] C. R. Hargraves and S. W. Paris, Direct Trajectory Optimization Using Nonlinear Programming and Collocation, Journal of Guidance, Control and Dynamics, Vol.10, pp.338-342, 1987.

[20] A. M. Hawkins, T. R. Fill, R. J. Proulx, E. M. Feron, Constrained Trajectory Optimization for Lunar Landing, AAS Spaceflight Mechanics Meeting, Tampa, FL, January 2006, AAS 06-153.

[21] S. I. Infeld and W. Murray, Optimization of stationkeeping for a Libration point mission, AAS Spaceflight Mechanics Meeting, Maui, HI, February 2004. AAS 04-150.

[22] W. Kang, M. Ross, and Q. Gong, Pseudospectral Optimal Control and Its Convergence Theorems, Lecture Notes in Control and Information Sciences, Alessandro Astolfi and Lorenzo Marconi eds., Springer, to appear.

[23] W. Kang, Q. Gong and I. M. Ross, Convergence of pseudospectral methods for nonlinear optimal control problems with discontinuous controller, 44th IEEE Conference on Decision and Control and European Control Conference (CDC-ECC'05), pp. 2799-2804, Seville, Spain, 2005.

[24] W. Kang, Q. Gong, I. M. Ross and F. Fahroo, On the convergence of nonlinear optimal control using pseudospectral methods for feedback linearizable control systems, to appear in International Journal on Robust and Nonlinear Control, 2007.

[25] W. Kang, On the Existence and Convergence of Solutions for Legendre Pseudospectral Optimal Control, preprint.

[26] L. R. Lewis, Rapid motion planning and autonomous obstacle avoidance for Unmanned Vehicles, Master's Thesis, Naval Postgraduate School, December 2006.

[27] P. Lu, H. Sun and B. Tsai, Closed-Loop endoatmospheric ascent guidance, Journal of Guidance, Control and Dynamics, Vol. 26, No. 2, pp. 283-294, 2003.

[28] B. S. Mordukhovich, Variational Analysis and Generalized Differentiation, I: Basic Theory, vol. 330 of Grundlehren der Mathematischen Wissenschaften [Fundamental Principles of Mathematical Sciences] Series, Springer, Berlin, 2005

[29] S. W. Paris and C. R. Hargraves, OTIS 3.0 Manual, Boeing Space and Defense Group, Seattle, WA, 1996.

[30] E. Polak, Optimization: Algorithms and Consistent Approximations, Springer-Verlag, Heidelberg, 1997.

[31] J. Rea, Launch vehicle trajectory optimization using a Legendre pseudospectral method, Proceedings of the AIAA Guidance, Navigation and Control Conference, Austin, TX, Paper No. AIAA 2003-5640, August 2003.

[32] I. M. Ross, Q. Gong, F. Fahroo and W. Kang, Practical stabilization through real-time optimal control, Proc. of American Control Conference, pp. 304-309, Minneapolis, MN, June, 2006.

[33] I. M. Ross, A historical introduction to the covector mapping principle, Advances in the Astronautical Sciences: Astrodynamics 2005, Vol. 122, Paper AAS 05-332, August 2005.

[34] I. M. Ross, User's manual for DIDO: A MATLAB application package for solving optimal control problems, Elissar LLC., Technical Report TR-705, 2007

[35] I. M. Ross and F. Fahroo, A unified framework for real-time optimal control, Proceedings of the IEEE Conference on Decision and Control, Maui, December, pp. 2210-2215, 2003.

[36] I. M. Ross and C. N. D'Souza, Hybrid Optimal Control Framework for Mission Planning, J. of Guid., Contr. and Dyn., Vol. 28, No. 4 pp. 686-697, 2005.

[37] I. M. Ross, P. Sekhavat, A. Fleming and Q. Gong, Pseudospectral feedback control: foundations, examples and experimental results, AIAA Guidance, Navigation, and Control Conference, AIAA-20066354, Keystone, Colorado, Aug., 2006, also to appear in AIAA Journal of Guidance, Control and Dynamics, 2007.

[38] I. M. Ross and F. Fahroo, Pseudospectral knotting methods for solving optimal control problems, AIAA Journal of Guidance, Control and Dynamics, Vol. 27, No. 3, pp. 397-405, 2004.

[39] I. M. Ross and F. Fahroo, A perspective on methods for trajectory optimization, Proceedings of the AIAA/AAS Astrodynamics Conference, Monterey, CA, August 2002. AIAA Paper No. 2002-4727.

[40] I. M. Ross and F. Fahroo, A pseudospectral transformation of the covectors of optimal control systems, Proceedings of the First IFAC Symposium on System Structure and Control, Prague, Czech Republic, 29-31 August 2001.

[41] I. M. Ross and F. Fahroo, Pseudospectral methods for optimal motion planning of differentially flat systems, IEEE Transactions on Automatic Control, Vol. 49, No. 8, pp. 1410-1413, August 2004.

[42] P. Sekhavat and I. M. Ross, On Closed-Loop Spacecraft Attitude Maneuvers, AAS Astrodynamics Specialist Conference, Mackinac Island, MI, August 2007, AAS 07-354.

[43] P. Sekhavat, A. Fleming and I. M. Ross, Time-Optimal Nonlinear Feedback Control for the NPSAT1 Spacecraft, Proceedings of the 2005 IEEE/ASME International Conference on Advanced Intelligent Mechatronics, AIM 2005, 2428 July 2005 Monterey, CA.

[44] S. Stanton, R. Proulx and C. N. D'Souza, Optimal orbit transfer using a Legendre pseudospectral method, AAS/AIAA Astrodynamics Specialist Conference, AAS-03-574, Big Sky, MT, August 3-7, 2003.

[45] L. N. Trefethen, Spectral Methods in MATLAB, SIAM, Philadelphia, PA, 2000.

[46] General Atomics Aeronautical Systems, "Aircraft Platforms: Altair," [http://www.ga-asi.com/products/index.php], 26 October 2006.

[47] V. M. Veliov, On the Time-discretization of Control Systems, SIAM J. Control Optim., Vol. 35, No. 5, pp. 1470-1486, 1997.

[48] P. Williams, C. Blanksby and P. Trivailo, Receding horizon control of tether system using quasilinearization and Chebyshev pseudospectral approximations, AAS/AIAA Astrodynamics Specialist Conference, Big Sky, MT, August 3-7, 2003, Paper AAS 03-535.

[49] H. Yan and K. T. Alfriend, Three-axis Magnetic Attitude Control Using Pseudospectral Control Law in Eccentric Orbits, AAS Spaceflight Mechanics Meeting, Tampa, FL, January 2006, AAS 06-103. 